\title{
Intermédialités
}

Histoire et théorie des arts, des lettres et des techniques

Intermediality

History and Theory of the Arts, Literature and Technologies

\section{Notices biobibliographiques}

Numéro 7, printemps 2006

URI : https://id.erudit.org/iderudit/1005660ar

DOI : https://doi.org/10.7202/1005660ar

Aller au sommaire du numéro

Éditeur(s)

Centre de recherche sur l'intermédialité

ISSN

1705-8546 (imprimé)

1920-3136 (numérique)

Découvrir la revue

Citer ce document

(2006). Notices biobibliographiques. Intermédialités / Intermediality, (7),

221-223. https://doi.org/10.7202/1005660ar d'utilisation que vous pouvez consulter en ligne.

https://apropos.erudit.org/fr/usagers/politique-dutilisation/ 


\section{Notices biobibliographiques Biobibliographical Notes}

Cécile Camart, diplômée en droit, en histoire de l'art et en muséologie, enseigne les arts contemporains et l'histoire de la photographie à l'Université de Provence (Aix-Marseille I). Elle collabore avec plusieurs revues (Artpress, Les cahiers du Musée national d'art moderne, Critique d'art, Photos nouvelles, Foam Magazine, ETC, Esse) et préface des monographies d'artistes (Katharina Bosse, Lucile Risch). Commissaire de l'exposition Sophie Calle au Ludwig Forum für Internationale Kunst (Aachen, Allemagne, 2005), elle a coédité en 2003 le catalogue Sophie Calle, M'as-tu vue (Paris, Éditions du Centre Pompidou / Éditions Xavier Barral). Sa thèse de doctorat en histoire de l'art (Rennes 2) a pour titre L'art de vivre selon Sophie Calle : esthétique de la situation, autofiction et mythologie en révolution. Elle a publié plusieurs articles sur l'artiste, dont «Sophie Calle, 19781981. Genèse d'une figure d'artiste », Les cahiers du Musée national d'art moderne, $\mathrm{n}^{\circ} 85$, automne 2003 et «Sophie Calle: de dérives en filatures: un érotisme de la séparation », Esse, n 55, automne 2005.

Martine Delvaux est professeure au Département d'études littéraires de l'UQAM. Elle est l'auteure de Femmes psychiatrisées femmes rebelles. De l'étude de cas à la narration autobiographique, Paris, Les empêcheurs de penser en rond, 1998; Ventriloquies, en collaboration avec Catherine Mavrikakis, Montréal, Leméac, 2003 ; et Histoires de fantômes. Spectralité et témoignage dans les récits de femmes contemporains, Montréal, Presses de l'Université de Montréal, 2005. Elle travaille entre autres sur le rapport texte/photo dans des ouvrages d'artistes et d'écrivaines contemporaines.

Arlette Farge est directrice de recherche au CNRS et enseignante à l'École des hautes études en sciences sociales (EHESS). Elle a dirigé le troisième volume (avec M. Davis) de L'histoire des femmes (G. Duby, M. Perrot, 1991) et elle est spécialiste des comportements et des sensibilités populaires au XVIII ${ }^{\mathrm{e}}$ siècle. Elle a notamment publié Le goût de l'archive (Seuil, 1991); Le cours ordinaire des 
choses dans la cité du XVIII siècle (Seuil, 1994) et a écrit avec Michel Foucault Le désordre des familles (Gallimard, 1982). À propos de la photographie, elle a publié dans la collection «Fiction et Cie», La chambre à deux lits et le cordonnier de Tel-Aviv (Seuil, 2000).

Bertrand Gervais a publié des essais sur la lecture, la littérature américaine et l'imaginaire, de même que des romans, des récits et des nouvelles. Il est professeur titulaire et enseigne au Département d'études littéraires de l'Université du Québec à Montréal. Il s'intéresse au roman américain contemporain, aux nouvelles formes fictionnelles, ainsi qu'aux théories sur l'imaginaire et ses figures. Il est le directeur de Figura, le Centre de recherche sur le texte et l'imaginaire, ainsi que du NT2, le Laboratoire de recherches littéraires sur les nouvelles formes de textes et de fictions.

Johnnie Gratton est professeur au Trinity College de Dublin. Il est l'auteur de Expressivism: The Vicissitudes of a Theory in the Writing of Proust and Barthes (Legenda, 2000), et le codirecteur de Modern French Short Fiction (Manchester University Press, 1994), La nouvelle hier et aujourd'hui (L'Harmattan, 1997), Subject Matters: Subject and Self in French Literature from Descartes to the Present (Rodopi, 2000), The Art of the Project: Projects and Experiments in Modern French Culture (Berghahn Books, 2005), et L'œil écrit. Études sur les rapports entre texte et image, 1800-1940 (Slatkine, 2005). Il a publié des articles sur Barthes, Breton, Sophie Calle, Colette, Foucault, Modiano, Proust, Sarraute, Jean-Loup Trassard, parmi d'autres.

Cybelle McFadden Wilkens a obtenu son doctorat en études françaises à Duke University en 2005. Elle est professeure adjointe en visite au Georgia Institute of Technology où elle enseigne le théâtre et le cinéma français et dirige des cours de langues. Elle a notamment publié «Body, Text, and Language: Wittig’s Struggle For The Universal in Les Guérillères » (Women in French Studies, No. 12, 2004).

Catherine Mavrikakis est professeure au Département d'études françaises de l'Université de Montréal depuis 2003. Auparavant, elle a occupé un poste de professeure à Concordia University de 1993 à 2003. Elle travaille sur la place de la photographie dans l'«autofiction" (première et dernière photos) et sur la spectralité, la thanatographie à l'œuvre dans les récits de disparition. Elle s'intéresse également au processus créateur dans la théorie psychanalytique et dans le discours tenu par les écrivains. Elle a publié des récits: Deuils cannibales 
et mélancoliques (Éditions Trois, 2000), Ça va aller (Leméac, 2002), Fleurs de crachat (Léméac, 2005) et Ventriloquies, écrit avec Martine Delvaux (Leméac, 2002), ainsi que l'essai Condamner à mort (Presses de l'Université de Montréal, 2005).

Magal Nachtergael est agrégée de Lettres modernes et prépare sa thèse de doctorat à l'Université Paris VII-Denis-Diderot sur le récit-photo dans l'art et la littérature. Critique d'art, elle collabore à la revue Artpress et enseigne l'histoire de l'art contemporain à l'Université de Bordeaux III. Elle a récemment publié «Daniel Pflumm, entreprise en produits artistiques», Parachute, n 118, 2005 ; «Nadja. Images, désir et sacrifice», Postures, n 7, 2005; "L’image écrite, 50 années d'expérimentations », L'art même, n’ 27, 2005.

Maïté Snauwaert a été chercheure post-doctorale au Centre de recherche sur le texte et l'imaginaire Figura à l'UQAM en 2004-2005. Docteure en Littérature française, elle a réalisé sa thèse, L'effet-Duras ou l'oeuvre lieu commun. Pour une politique de la lecture, à l'Université Paris viII. Elle est l'auteure de: «Mise en récit / mise en série: le recyclage dans le dispositif de Douleur exquise de Sophie Calle », $\mathrm{n}^{\circ}$ 18, La voix du regard, 2006, et de plusieurs études sur Marguerite Duras, en particulier «Écrire de Marguerite Duras : une poétique de l'illisible », dans Ricard Ripoll (dir.), Stratégies de l'illisible, Presses universitaires de Perpignan, 2005, et "Histoire de livres et livre d'histoires: La pluie d'été», dans Alexandra Saemmer et Stéphane Patrice (dirs.), Les lectures de Marguerite Duras, Presses universitaires de Lyon, 2005. Elle est actuellement chercheure post-doctorale au Centre de recherche interdisciplinaire sur la littérature et la culture québécoise (CRILCQ) à l'Université de Montréal, où elle étudie l'impact du roman familial dans les littératures française et québécoise contemporaines.

Perin Emel Yavuz, doctorante à l'EHESS (Paris), prépare une thèse sur le Narrative art sous la direction de Jean-Marie Schaeffer. Elle participe régulièrement à des colloques internationaux et journées d'études, et publie des articles sur l'art contemporain dans une perspective esthétique et linguistique. Elle coordonne avec Séverine Sofio (EHESS) un numéro de la revue Les cahiers du genre sur l'art, le genre et la valeur. Elle a obtenu deux bourses de l'école française de Rome (2004) et du Graduiertenkolleg "Körper-Inszenierungen” - Freien Universität de Berlin (2006). 